\title{
Additional Value of Intra-Aneurysmal Hemodynamics in Discriminating Ruptured versus Unruptured Intracranial Aneurysms
}

J.J. Schneiders, H.A. Marquering, P. van Ooij, R. van den Berg, A.J. Nederveen, D. Verbaan, W.P. Vandertop, M. Pourquie, G.J.E. Rinkel, E. vanBavel, and C.B.L.M. Majoie

\begin{abstract}
BACKGROUND AND PURPOSE: Hemodynamics are thought to play an important role in the rupture of intracranial aneurysms. We tested whether hemodynamics, determined from computational fluid dynamics models, have additional value in discriminating ruptured and unruptured aneurysms. Such discriminative power could provide better prediction models for rupture.
\end{abstract}

\begin{abstract}
MATERIALS AND METHODS: A cross-sectional study was performed on patients eligible for endovascular treatment, including 55 ruptured and 62 unruptured aneurysms. Association with rupture status was tested for location, aneurysm type, and 4 geometric and 10 hemodynamic parameters. Patient-specific spatiotemporal velocities measured with phase-contrast MR imaging were used as inflow conditions for computational fluid dynamics. To assess the additional value of hemodynamic parameters, we performed 1 univariate and 2 multivariate analyses: 1 traditional model including only location and geometry and 1 advanced model that included patient-specific hemodynamic parameters.
\end{abstract}

RESULTS: In the univariate analysis, high-risk locations (anterior cerebral arteries, posterior communicating artery, and posterior circulation), daughter sacs, unstable inflow jets, impingements at the aneurysm body, and unstable complex flow patterns were significantly present more often in ruptured aneurysms. In both multivariate analyses, only the high-risk location (OR, 3.92; 95\% Cl, 1.77-8.68) and the presence of daughter sacs (OR, 2.79; $95 \% \mathrm{Cl}, 1.25-6.25)$ remained as significant independent determinants.

CONCLUSIONS: In this study population of patients eligible for endovascular treatment, we found no independent additional value of aneurysmal hemodynamics in discriminating rupture status, despite high univariate associations. Only traditional parameters (high-risk location and the presence of daughter sacs) were independently associated with ruptured aneurysms.

ABBREVIATIONS: $C F D=$ computational fluid dynamics; $3 \mathrm{DRA}=3 \mathrm{D}$ rotational angiography; $\mathrm{PC}=$ phase-contrast

$\mathrm{T}$ he prevalence of intracranial aneurysms in the general population is approximately $1 \%-5 \% .{ }^{1,2}$ Although most aneurysms remain asymptomatic, a minority rupture, and this scenario is associ-

Received September 10, 2014; accepted after revision March 3, 2015.

From the Departments of Radiology (J.J.S., H.A.M., P.v.O., R.v.d.B., A.J.N., C.B.L.M.M.), Biomedical Engineering and Physics (H.A.M., E.V.), and Neurosurgery (D.V., W.P.V.), Academic Medical Center, Amsterdam, the Netherlands; Department of Aero- and Hydrodynamics (M.P.), Delft University of Technology, Delft, the Netherlands; and Department of Neurology and Neurosurgery (G.J.E.R.), Rudolf Magnus Institute of Neuroscience, University Medical Centre Utrecht, Utrecht, the Netherlands. H.A. Marquering and P. van Ooij contributed equally.

This study was supported by a grant from the Foundation Nuts Ohra, Amsterdam, the Netherlands, and the Gratama Foundation, the Netherlands.

Please address correspondence to J.J. Schneiders, MD, PhD, Department of Neuroradiology, Cl-425, Meibergdreef 9, 1105 AZ Amsterdam, the Netherlands; e-mail: j.j.schneiders@amc.uva.nl

= Indicates article with supplemental on-line tables.

Indicates article with supplemental on-line photo.

Mvidence-Based Medicine Level 2.

http://dx.doi.org/10.3174/ajnr.A4397 ated with high morbidity and case fatality rates. ${ }^{2}$ For unruptured aneurysms, the risk of treatment complications has to be carefully balanced against the future risk of rupture. At present, risk assessment of unruptured intracranial aneurysms and the decision to treat or observe are mainly based on patient age, family history, aneurysm size, and location. ${ }^{3-5}$ However, the predictive value of these characteristics is limited. For example, most ruptured aneurysms are smaller than the recommended minimum of $7 \mathrm{~mm}$ for treatment. ${ }^{6}$

Several researchers have attempted to better stratify rupture risk by assessments of the associations between local hemodynamic features and aneurysm formation, growth, and rupture by using computational fluid dynamics (CFD). ${ }^{7-10}$ In general, due to the difficulty of obtaining patient-specific velocity measurements, assumptions are made for the inflow boundary conditions. However, several studies have shown a large interpatient variation of intracranial artery flow. ${ }^{11,12}$

The purpose of this study was to test whether estimation of local hemodynamics has additional value in discriminating ruptured and unruptured aneurysms. Thus, high-resolution 3D 


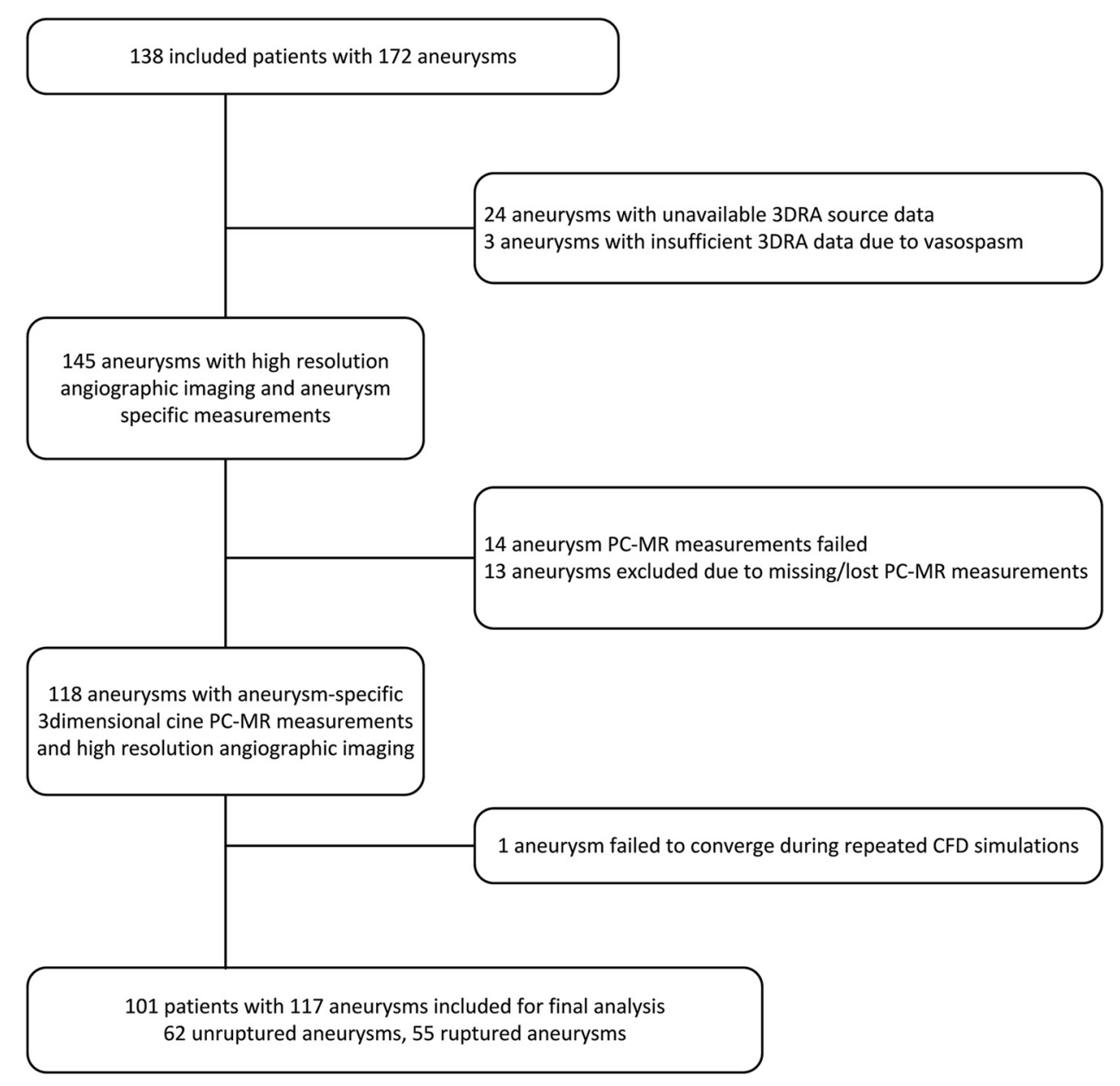

FIG 1. Flow chart of patient and aneurysm inclusion.

geometry and patient-specific measurements of local flow velocities in the afferent artery as boundary conditions for hemodynamic simulations were used. ${ }^{13-15}$

\section{MATERIALS AND METHODS}

Patients with intracranial aneurysms were invited to participate in this study by a consulting neurointerventionalist during a visit at the outpatient clinic in our tertiary care referral center from January 2009 to October 2011. Patients with ruptured aneurysms were invited to participate at their first consultation after discharge from the hospital. Patients with unruptured aneurysms were asked to participate during their work-up for aneurysm treatment. Inclusion criteria were the following: aneurysm size of $>3 \mathrm{~mm}, 18-75$ years of age, and the ability to give informed consent. Exclusion criteria were contraindications for 3T MR imaging and previous aneurysm clipping. One hundred thirty-eight patients (with 172 aneurysms) agreed to participate. The study was approved by the institutional review board. Written informed consent was obtained from all patients.

We excluded 55 aneurysms ( 37 patients) for the following reasons: missing 3D rotational angiography (3DRA) source data ( $n=24)$; insufficient quality of 3DRA due to vasospasm $(n=3)$; missing $(n=13)$ or failed $(n=14)$ phase-contrast MR imaging (PC-MR imaging) measurements due to inaccurate cardiac gating; or failed CFD simulation $(n=1)$ (Fig 1$)$.
The location of the ruptured or unruptured aneurysms was determined (Table). Because aneurysms located at the anterior cerebral arteries (anterior communicating artery, anterior cerebral artery, pericallosal artery), posterior communicating artery, and in the posterior circulation (basilar artery, vertebral artery, cerebellar arteries) are associated with a higher risk of rupture, ${ }^{4}$ dichotomization was performed by grouping these high-risk locations and comparing them with the other, lower risk locations.

In patients with multiple aneurysms with 1 rupture (6 patients), 2 experienced neuroradiologists (C.B.L.M.M., R.v.d.B., both with $>10$ years of experience) inspected all clinical and radiologic data in consensus to select the most likely source of hemorrhage. The other aneurysms were classified as unruptured.

\section{Imaging Protocols}

Acquisition of 3DRA was part of the standard clinical work-up. Images were acquired with either local (patients with unruptured aneurysms) or general anesthesia (during endovascular treatment in patients with ruptured aneurysms) by using a single-plane angiographic unit (Integris Allura Neuro; Philips Healthcare, Best, the Netherlands) following institutional protocol. 3DRA was acquired during a 6-second run, with $21 \mathrm{~mL}$ of contrast (iodixanol, Visipaque; GE Healthcare, Piscataway, New Jersey), which was administered at $3 \mathrm{~mL} / \mathrm{s}$, resulting in a $256^{3}$ isotropic image volume. The 3DRA was started 3 seconds after contrast injection. 
Location, aneurysm type, and rupture status of 117 aneurysms (101 patients)

\begin{tabular}{|c|c|c|c|c|c|c|c|}
\hline \multirow[b]{2}{*}{ Location $^{a}$} & \multirow[b]{2}{*}{ No. of Cases } & \multicolumn{2}{|c|}{ Total $(n=117)$} & \multicolumn{2}{|c|}{ Bifurcation $(n=90)$} & \multicolumn{2}{|c|}{ Sidewall $(n=27)$} \\
\hline & & Unruptured & Ruptured & Unruptured & Ruptured & Unruptured & Ruptured \\
\hline \multicolumn{8}{|c|}{ High-risk location } \\
\hline PcomA & $12(10 \%)$ & 4 & 8 & 3 & 3 & 1 & 5 \\
\hline BA & $11(9 \%)$ & 8 & 3 & 8 & 2 & 0 & 1 \\
\hline VA & $1(1 \%)$ & 0 & 1 & 0 & 0 & 0 & 1 \\
\hline ACA & $8(7 \%)$ & 3 & 5 & 2 & 5 & 1 & 0 \\
\hline AcomA & $28(24 \%)$ & 7 & 21 & 7 & 20 & 0 & 1 \\
\hline Subtotal & $60(51 \%)$ & 22 & 38 & 20 & 30 & 2 & 8 \\
\hline \multicolumn{8}{|c|}{ Low-risk location } \\
\hline MCA & $36(31 \%)$ & 27 & 9 & 23 & 9 & 4 & 0 \\
\hline ICA & $21(18 \%)$ & 13 & 8 & 3 & 5 & 10 & 3 \\
\hline Subtotal & 57 (49\%) & 40 & 17 & 26 & 14 & 14 & 3 \\
\hline Total & 117 (100\%) & 62 & 55 & 46 & 44 & 16 & 11 \\
\hline
\end{tabular}

Note:-AcomA indicates anterior communicating artery; PcomA, posterior communicating artery; VA, vertebral artery.

a ICA includes the internal carotid, ophthalmic, anterior choroidal arteries and the carotid tip; ACA includes the anterior cerebral and pericallosal arteries; BA includes the basilar, PICA, and superior cerebellar arteries.

MR imaging was performed at 3T (Intera; Philips Healthcare) and included a multiple overlapping thin-slab-acquisition $3 \mathrm{D}$ TOF MRA sequence and a 3D PC-MR imaging sequence with heart rate monitoring by electrocardiography or by a Peripheral Pulse Unit (Siemens, Erlangen, Germany) for velocity measurements in the afferent artery proximal to the aneurysm. The multiple overlapping thin-slab-acquisition 3D TOF had a scan resolution of $0.39 \times 0.6 \times 1 \mathrm{~mm}$. Other parameters were the following: TE/TR/flip angle, $4.2 / 21.4 \mathrm{~ms} / 20^{\circ}$; parallel imaging factor, 2.5; scanning time, 6 minutes and 16 seconds.

3D PC-MR imaging (time-resolved measurement of velocity in 3 directions in a single plane perpendicular to the artery) was performed with a resolution of $0.64 \times 0.65 \times 3 \mathrm{~mm}$; TE/TR/flip angle, $5.7 / 8.5 \mathrm{~ms} / 10^{\circ}$; receiver bandwidth, $172 \mathrm{kHz}$; parallel imaging factor, 2. Velocity-encoding was $100 \mathrm{~cm} / \mathrm{s}$ and could be adjusted to $70 \mathrm{~cm} / \mathrm{s}$ if needed. The velocity information was acquired in 23-36 cardiac phases. We ensured that the location of the 3D PC-MR imaging measurement was distal to any proximal branching artery $(>1 \mathrm{~mm})$ to obtain the most accurate velocity and flow information.

Patients with unruptured aneurysms were asked to undergo an additional PC-MR imaging study before endovascular treatment. In patients with ruptured aneurysms who were treated in an acute setting, it was not possible to perform PC-MR imaging velocity measurements before intervention, and these data were obtained at follow-up 6 months after coiling. All unruptured aneurysms were compared with previous imaging to detect possible geometric changes during the time interval of 3DRA and MR imaging. Postprocessing of the MR images was described in detail previously (On-line Fig). ${ }^{16}$

\section{Vascular Models and Vascular Model Correction}

A level set algorithm was used to segment the vascular tree in 3DRA image data. ${ }^{17}$ The segmentations were converted into a tetrahedral element mesh with $>1,000,000$ tetrahedral elements $\left( \pm 3000\right.$ elements $\left.\times \mathrm{mm}^{-3}\right)$. Neck size overestimation was reviewed by 2 neuroradiologists, by comparing the segmentations with those in 2D DSA. Twenty-six of 117 aneurysm segmentations showed neck size overestimation and required modification according to a previously described method. ${ }^{18}$

\section{Hemodynamic Modeling and Visualization}

Fluent 6.3 (ANSYS, Canonsburg, Pennsylvania) was used to simulate hemodynamics. In-house-developed software was used to impose PC-MR imaging-measured spatiotemporal velocity profiles as inflow boundary conditions. ${ }^{16}$ In case of anterior communicating artery aneurysms, DSA images were reviewed by 2 neuroradiologists to determine the afferent artery. None of the anterior communicating artery aneurysms filled from both A1s.

The outflow ratio of the distal arteries was calculated by using the Murray law. A no-slip boundary was set at the vessel wall, and rigid walls were assumed. ${ }^{19}$ Density and dynamic viscosity were, respectively, $1060 \mathrm{~kg} \times \mathrm{m}^{-3}$ and $0.004 \mathrm{~kg} \times$ $\mathrm{m}^{-1} \times \mathrm{s}^{-1}$. Three complete cardiac cycles were calculated; the third cycle was used for analysis. The flow patterns resulting from the CFD simulations were visualized with streamlines and isosurfaces as movie clips by using Paraview 3.6 (Kitware; Los Alamos National Laboratory and Sandia National Laboratories, Los Alamos, New Mexico) (Fig 2).

\section{Quantitative Parameter Assessment}

The following geometric aneurysm parameters were determined from the 3D models by a research physician (J.J.S.): size (largest diameter in millimeters), ${ }^{20}$ aspect ratio (height divided by the neck size), ${ }^{21}$ and spheric or nonspheric nature of the aneurysm ("spheric" defined as an aneurysm height within $80 \%-125 \%$ of its width). ${ }^{20}$ Quantitative hemodynamic parameters (mean wall shear stress, maximum wall shear stress, and oscillatory shear index) were derived from the hemodynamic data provided by CFD by using in-house-developed software. The quantitative hemodynamic parameters were derived from the surface of the aneurysm sac. Time-dependent indices (oscillatory shear index) were calculated with a time-averaged method by using all the cardiac phases available.

\section{Qualitative Parameter Assessment}

Qualitative parameters were assessed by 2 interventional neuroradiologists (C.B.L.M.M., R.v.d.B.). Qualitative geometric parameters were assessed from the $3 \mathrm{D}$ model and included aneurysm type (sidewall or bifurcation) and the presence or absence of 


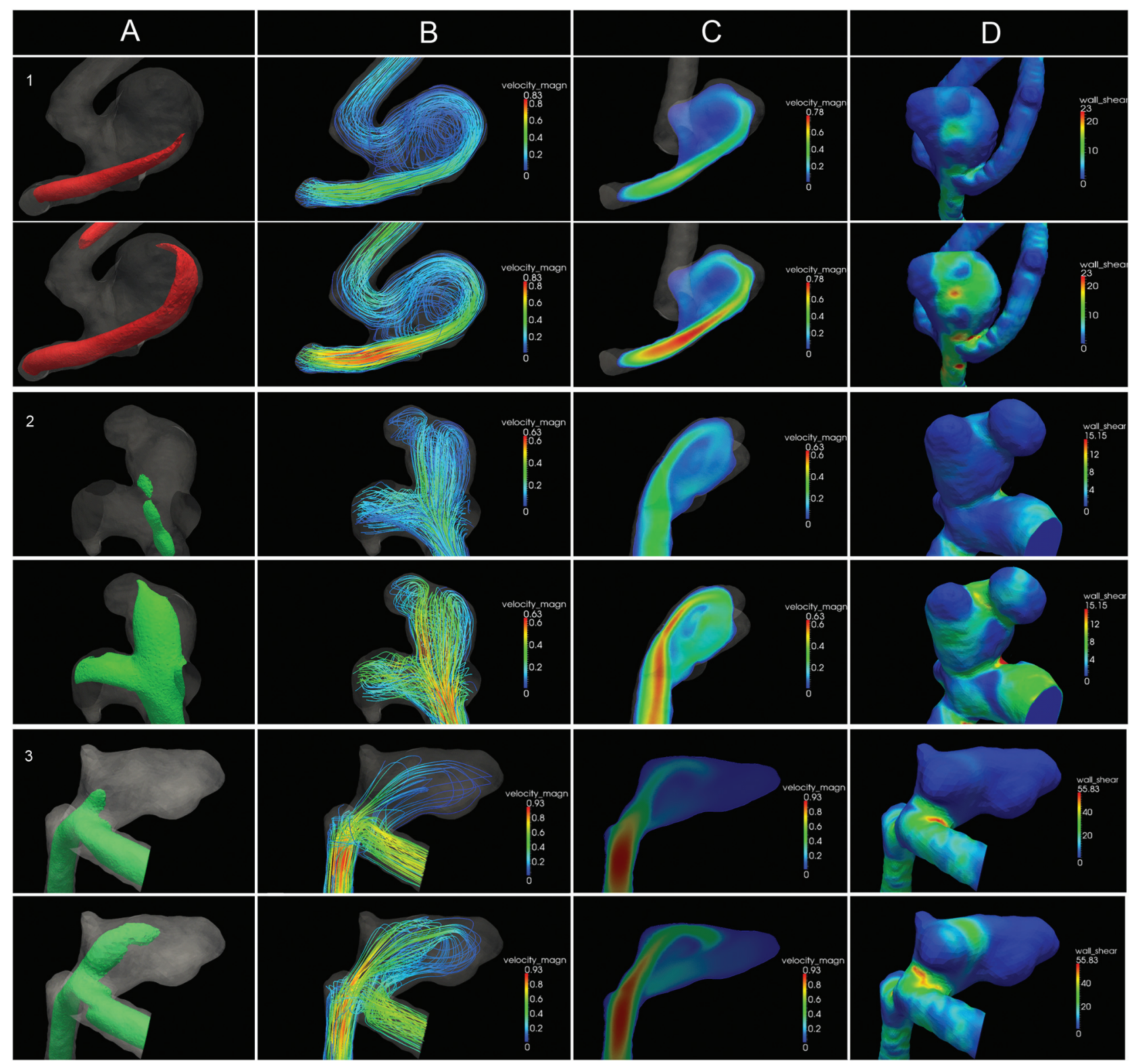

FIG 2. Intra-aneurysmal hemodynamics in 3 typical aneurysms. Diastole is depicted in the top row; systole, in the bottom row. The columns represent: $A$, Isosurface of the velocity. $B, 3 D$ streamlines colored by velocity magnitude. $C, 2 D$ slice of the velocity magnitude. $D$, Wall shear stress distribution displayed on 3D geometry. The rows represent cases 1-3. Case 1: Ruptured anterior communicating artery aneurysm with a concentrated inflow jet $(A$ and $C$ ) and a stable-simple flow pattern: single vortex that is stable throughout the cardiac cycle $(B)$. The impingement zone is located at the body $(D)$. Case 2: Unruptured posterior communicating artery aneurysm with a concentrated inflow jet $(A$ and $C)$ and a stable-complex flow pattern: multiple flow structures that remain stable throughout the cardiac cycle $(B)$. The impingement zone is located at the dome $(D)$ and 2 daughter sacs $(D)$. Case 3: Ruptured anterior communicating artery aneurysm with a concentrated inflow jet $(A$ and $C)$ and an unstable-simple flow pattern: a single flow structure that is unstable throughout the cardiac cycle. The primary direction of flow is different between systole and diastole (B). The impingement zone is located at the neck $(D)$ and 2 daughter sacs $(D)$.

daughter sacs. An aneurysm was classified as a sidewall aneurysm when it originated from a major vessel trunk with either no or a very small side branch (with a diameter of less than one-fifth of the parent vessel). ${ }^{10}$ All other aneurysms were classified as bifurcation aneurysms.

Qualitative hemodynamic parameters were assessed from movie clips and included the number of inflow jets (single or multiple), inflow jet concentration (concentrated or diffuse), inflow jet stability (stable or unstable), vortex complexity (simple or complex), vortex stability (stable or unstable) flow pattern category according to Cebral et al, ${ }^{9}$ and location of the impingement zone.
The inflow jet was considered unstable if its main direction changed throughout the cardiac cycle. The inflow jet was considered concentrated if the inflow width was less than half the aneurysm neck. Vortices were assessed for complexity (simple/complex) and stability (stable/unstable). Vortices were considered complex if there was $>1$ major flow structure; they were categorized as unstable when $\geq 1$ flow structure disappeared during a cardiac cycle. The stability and complexity of intra-aneurysmal flow were classified by assigning 1 of the following 4 flow types: 1 ) stable-simple: stable direction of the inflow jet with a single associated vortex, 2) stable-complex: stable direction of the inflow jet 
with multiple associated vortices and no change in the number of vortices during the cardiac cycle, 3) unstable-simple: changing direction of inflow jet with the creation of a single vortex, and 4) unstable-complex: changing direction of the inflow jet associated with the creation or destruction of multiple vortices. ${ }^{9}$

Images of the wall shear stress magnitude were used to categorize the location of the impingement zone as neck, body, dome, or in a daughter sac. ${ }^{19}$ The "impingement zone" was defined as the area on the aneurysm sac where the inflow jet deflects.

\section{Statistical Analysis}

The Shapiro-Wilk test was used to assess the normality of the distribution of continuous variables (normally distributed if the $W$ statistic was $>0.9$ ). The normally distributed variable (size) was expressed as mean with $\mathrm{SD}$, whereas not normally distributed variables were presented as medians with interquartile ranges (interquartile range, $25 \%-75 \%$ ).

To evaluate the relation between determinants and rupture status, we performed univariate and multivariate analyses by using logistic regression analysis. Variables that were significantly associated with rupture status in the univariate analysis were included in the multivariate analysis by using manual backward variable selection. To test for multicollinearity, we calculated the variance inflation factor. We generated 2 multivariate models: a "traditional" model including only the location and geometric determinants, and an advanced model including location and geometric and hemodynamic determinants. Interobserver agreement of all parameters was assessed by calculation of the intraclass correlation coefficient and the percentage of agreement. A $P$ value $<.05$ was considered statistically significant in all analyses. All analyses were performed by using the SPSS, Version 20.0 (IBM, Armonk, New York).

\section{RESULTS}

In the analysis, 101 patients (63 women; mean age, 54.8 years) with 117 aneurysms (62 unruptured, 55 ruptured) were included (Fig 1). The distribution of aneurysm locations is depicted in the Table. The unruptured aneurysms had a mean size of $7.4 \pm 3.7$ $\mathrm{mm}$; the ruptured aneurysms, $6.6 \pm 2.8 \mathrm{~mm}$ (On-line Table 1). Eleven of the included patients had multiple aneurysms: 7 patients with 2 aneurysms, 3 with 3 aneurysms, and 1 with 4 aneurysms. Multiple aneurysms per patient were included if specific PC-MR imaging measurements were available for each aneurysm. None of the unruptured aneurysms showed geometric changes during the interval between 3DRA and MR imaging.

The univariate associations with aneurysm rupture status are depicted in On-line Table 1. There was no indication of severe multicollinearity. The interobserver agreement for qualitatively scored hemodynamic parameters is displayed in On-line Table 2. Aneurysms located at high-risk locations were more often associated with rupture compared with the aneurysms at other locations (OR, 4.06; 95\% CI, 1.88-8.81). The only geometric parameter that was significantly associated with rupture status was the presence of a daughter sac (OR, 2.93; 95\% CI, 1.37-6.29). Regarding the hemodynamic parameters, unstable inflow jet (OR, 13.56; 95\% CI, 1.67-110), impingement zone location at the aneurysm body (OR, 2.49; 95\% CI, 1.00-6.19), and complex vortices (OR,
2.34; 95\% CI, 1.11-4.96) were significantly associated with ruptured aneurysms. In addition, an unstable-complex flow pattern was present significantly more often in ruptured aneurysms (OR, 2.59; 95\% CI, 1.05-6.37) compared with a stable-simple flow pattern. The presence of stable-complex and unstable-simple flow patterns was not different in ruptured and unruptured aneurysms.

In the traditional multivariate model, both high-risk location (OR, 3.92; 95\% CI, 1.77-8.68) and the presence of daughter sacs (OR, 2.79; 95\% CI, 1.25-6.25) were independently associated with ruptured aneurysms (Nagelkerke $R^{2}$ of 0.21 ; area under the curve, $0.72 ; 95 \% \mathrm{CI}, 0.63-0.82)$. The advanced multivariate model added (univariate significant) hemodynamic parameters: unstable inflow jet, vortex complexity, flow-pattern category, and location of the impingement zone. In this advanced model, highrisk location and the presence of daughter sacs were independently associated with ruptured aneurysms (OR values equal to the traditional model), resulting in exactly the same area under the curve (area under the curve, 0.72; 95\% CI, 0.63-0.82; Nagelkerke $R^{2}$ of 0.21 ). Thus, hemodynamic parameters did not improve the traditional multivariate model.

\section{DISCUSSION}

In this study, which compared geometric and hemodynamic parameters in 117 aneurysms, no additional value of aneurysmal hemodynamics for the characterization of ruptured-versus-unruptured aneurysms was found. Rather, only the presence of daughter sacs and location at the anterior cerebral arteries, posterior communicating artery, or posterior circulation significantly characterized ruptured aneurysms, and this was the case for multivariate models with or without inclusion of hemodynamics.

Various hemodynamic aspects of aneurysms have been implicated as potential predictors of the risk of rupture, such as disturbed flow patterns, small impingement regions, narrow jets, wall shear stress, and oscillatory shear index. ${ }^{9,10,22}$ In addition, these complex and unstable flow patterns have been correlated with a clinical history of prior aneurysm rupture. ${ }^{8}$ This study does not address the causality of hemodynamics and aneurysm rupture and does not allow any statements considering causality. While univariate analyses in the current work and previous studies clearly argue for hemodynamic data as discriminators and candidate predictors for rupture, our multivariate models do not show additional value when we account for geometric data.

The independent association of location at the anterior cerebral arteries, posterior communicating artery, and posterior circulation with rupture is in accordance with a recently published pooled analysis based on 6 prospective studies with 8382 patients with unruptured aneurysms. ${ }^{4}$ That analysis, however, did not include hemodynamic parameters. Despite the evidence, several studies on hemodynamic risk factors for aneurysm rupture did not include location as a determinant in their analyses. ${ }^{8,10}$ A post hoc analysis of the findings of Xiang et $\mathrm{al}^{10}$ indicates that also in that study, location at the anterior cerebral arteries, posterior communicating artery, and posterior circulation is positively associated with rupture, with $84 \%$ in the ruptured group, as opposed to $33 \%$ in the unruptured group. It is not clear whether the 
shear parameters would remain significantly involved if location had been included in their model.

Daughter sacs were present significantly more often in ruptured aneurysms compared with unruptured aneurysms. The importance of aneurysm shape for the risk profile has previously been acknowledged in a study comparing ruptured and unruptured cerebral aneurysms, in which $10 \%$ of unruptured aneurysms showed a multilobular appearance on DSA compared with $20 \%$ of ruptured aneurysms. ${ }^{23}$ In the Unruptured Cerebral Aneurysm Study of Japan, daughter sacs were also associated with an increased rupture rate. ${ }^{24}$ Aneurysm geometry and the presence of daughter sacs may change before, during, or after rupture. Two studies on 13 and 9 patients before and after rupture showed that indeed geometry after rupture may be different due to growth and displacements by hematoma. ${ }^{25,26}$ While rupture might have affected the percentage of aneurysms with visible daughter sacs, the Unruptured Cerebral Aneurysm Study of Japan underlines the view that the significant association in our study with ruptured aneurysms is not merely a consequence of rupture.

Aneurysm size did not significantly contribute to characterization of the rupture state, while it is a proved risk factor for aneurysm rupture in longitudinal follow-up studies. ${ }^{3,5,24}$ The reason for this finding is that in patients with ruptured aneurysms, 3DRA was routinely performed in the standard work-up protocol in all aneurysms regardless of size. However, patients with unruptured aneurysms were only scheduled for treatment when aneurysm size was $>7 \mathrm{~mm}$ or when aneurysm growth was seen during follow-up. Unruptured MCA aneurysms were over-represented in our study compared with the patient population of the institution, though this number was comparable with that in a study by Morita et al. ${ }^{24}$ This difference might have been related to the more frequent application of 3DRA imaging in relation to decisions for treatment.

In a recent study of Baharoglu et $\mathrm{al},{ }^{27}$ rupture risk factors were assessed for sidewall and bifurcation aneurysms separately. This approach is promising for dealing with multicollinearity. The design and number of included patients in this study did not allow us to also compare all variables against these 2 aneurysm types.

Patient-specific inflow patterns for CFD, based on PC-MR imaging, were not always obtained within a short interval of 3DRA. Patients with unruptured aneurysms were asked to participate right before or after 3DRA, either in the pretherapeutic work-up or during an elective coiling procedure. Patients with a recent 3DRA ( $<1$ year) were asked to participate during regular checkups at the outpatient clinic. For ruptured aneurysms, PC-MR imaging was performed 6 months after treatment. We do not believe that these timing differences biased the estimation of hemodynamic patterns. Cebral et $\mathrm{al}^{19}$ indicated that for variation of inflow within a $\sim 25 \%$ range, calculated flow patterns remain unchanged. In the absence of major events such as rupture or stroke, no such variation is expected.

Data on flow changes after coiling are sparse. However, in a pilot study, we found that flow changes in inlet and outlet vessels before and after coiling are within this $25 \%$ range. These results, therefore, allow the PC-MR imaging flow measurements performed 6 months after coiling to serve as a boundary condition for CFD simulating pretreatment hemodynamics.
Several assumptions for the CFD simulations had to be made, including traction-free boundary conditions, rigid walls, and outlet boundary conditions. However, these assumptions are believed to have relatively limited influence on the predicted flow patterns. ${ }^{28}$ The influence of mesh resolution is reported in an article by Valen-Sendstad and Steinman, ${ }^{29}$ in which they have shown that lower resolution meshes can result in different oscillatory shear index values as opposed to higher resolution models. Not using the very-high-resolution models may have disguised the associations between oscillatory shear index and rupture status; however, this issue was beyond the scope of this study.

A limitation of this study is the absence of other established clinical risk factors for rupture, including multiple aneurysms, ${ }^{30}$ a history of previous $\mathrm{SAH},{ }^{31}$ hypertension, ${ }^{4}$ female sex, ${ }^{5}$ and descent. ${ }^{5}$ Including such factors was not possible because it would have affected the power of tests for the hemodynamic parameters. It remains to be established whether such clinical factors interact with hemodynamics in risk prediction.

We reported the interobserver agreement for the classifications of our experts before consensus was reached. The agreement of the variables flow concentration, inflow stability, and flow complexity is, on average, $85 \%$, compared with $99 \%$ in a previous study. ${ }^{8}$ The lower agreement and relatively low intraclass correlation coefficient for most variables may reduce the discriminating power of the analysis. Therefore, in the final analysis, we used the consensus of the 2 observers for all scored variables.

The current cross-sectional design does not allow straightforward estimation of rupture risk from the included parameters. For example, unruptured aneurysms included in this study may still have a high risk for future rupture, and this study cannot discriminate between aneurysms that have not yet ruptured and those that will never rupture.

The lack of additional value of hemodynamic parameters raises the question of whether and how such hemodynamics could be part of risk of rupture models. Larger longitudinal studies will provide more insight than could possibly be generated in the current cross-sectional design.

\section{CONCLUSIONS}

In this study population of patients eligible for endovascular treatment, we found no independent additional value of aneurysmal hemodynamics in discriminating rupture status, despite high univariate associations. Only traditional parameters (high-risk location and the presence of daughter sacs) were independently associated with ruptured aneurysms.

Disclosures: Ed vanBavel—UNRELATED: Grants/Grants Pending: Marie Curie Program of the European Union. * Charles B.L.M. Majoie—RELATED: Grant: Nuts Ohra Foundation,* Comments: SNO-T-0702-92; a research grant to evaluate the additional value of hemodynamics on the rupture risk assessment in intracranial aneurysms, * Gratama Foundation*; UNRELATED: Grants/Grants Pending: Dutch Heart Foundation*; Payment for Lectures (including service on Speakers Bureaus): Stryker. * Money paid to the institution.

\section{REFERENCES}

1. Rinkel GJ, Djibuti M, Algra A, et al. Prevalence and risk of rupture of intracranial aneurysms: a systematic review. Stroke 1998;29:251-56

2. Brisman JL, Song JK, Newell DW. Cerebral aneurysms. N Engl J Med 2006;355:928-39

AJNR Am J Neuroradiol 36:1920-26 Oct 2015 www.ajnr.org 1925 
3. Wiebers DO, Whisnant JP, Huston J 3rd, et al; International Study of Unruptured Intracranial Aneurysms Investigators. Unruptured intracranial aneurysms: natural history, clinical outcome, and risks of surgical and endovascular treatment. Lancet 2003;362:103-10

4. Greving JP, Wermer MJ, Brown RD Jr, et al. Development of the PHASES score for prediction of risk of rupture of intracranial aneurysms: a pooled analysis of six prospective cohort studies. Lancet Neurol 2014;13:59-66

5. Wermer MJ, van der Schaaf IC, Algra A, et al. Risk of rupture of unruptured intracranial aneurysms in relation to patient and aneurysm characteristics: an updated meta-analysis. Stroke 2007;38:1404-10

6. Forget TR Jr, Benitez R, Veznedaroglu E, et al. A review of size and location of ruptured intracranial aneurysms. Neurosurgery 2001;49: 1322-25; discussion 1325-26

7. Miura Y, Ishida F, Umeda Y, et al. Low wall shear stress is independently associated with the rupture status of middle cerebral artery aneurysms. Stroke 2013;44:519-21

8. Cebral JR, Mut F, Weir J, et al. Association of hemodynamic characteristics and cerebral aneurysm rupture. AJNR Am J Neuroradiol 2011;32:264-70

9. Cebral JR, Castro MA, Burgess JE, et al. Characterization of cerebral aneurysms for assessing risk of rupture by using patient-specific computational hemodynamics models. AJNR Am J Neuroradiol 2005;26:2550-59

10. Xiang J, Natarajan SK, Tremmel M, et al. Hemodynamic-morphologic discriminants for intracranial aneurysm rupture. Stroke 2011;42:144-52

11. Schneiders JJ, Ferns SP, van Ooij P, et al. Comparison of phasecontrast MR imaging and endovascular sonography for intracranial blood flow velocity measurements. AJNR Am J Neuroradiol 2012;33:1786-90

12. Radaelli AG, Augsburger L, Cebral JR, et al. Reproducibility of haemodynamical simulations in a subject-specific stented aneurysm model: a report on the Virtual Intracranial Stenting Challenge 2007. J Biomech 2008;41:2069-81

13. Venugopal P, Valentino D, Schmitt H, et al. Sensitivity of patientspecific numerical simulation of cerebral aneurysm hemodynamics to inflow boundary conditions. J Neurosurg 2007;106:1051-60

14. Marzo A, Singh P, Larrabide I, et al. Computational hemodynamics in cerebral aneurysms: the effects of modeled versus measured boundary conditions. Ann Biomed Eng 2011;39:884-96

15. Jansen IG, Schneiders JJ, Potters WV, et al. Generalized versus patient-specific inflow boundary conditions in computational fluid dynamics simulations of cerebral aneurysmal hemodynamics. AJNR Am J Neuroradiol 2014;35:1543-48

16. van Ooij P, Schneiders JJ, Marquering HA, et al. 3D cine phase-contrast MRI at $3 \mathrm{~T}$ in intracranial aneurysms compared with patientspecific computational fluid dynamics. AJNR Am J Neuroradiol 2013;34:1785-91

17. Piccinelli M, Veneziani A, Steinman DA, et al. A framework for geo- metric analysis of vascular structures: application to cerebral aneurysms. IEEE Trans Med Imaging 2009;28:1141-55

18. Schneiders JJ, Marquering HA, Antiga L, et al. Intracranial aneurysm neck size overestimation with 3D rotational angiography: the impact on intra-aneurysmal hemodynamics simulated with computational fluid dynamics. AJNR Am J Neuroradiol 2013;34:121-28

19. Cebral JR, Castro MA, Appanaboyina S, et al. Efficient pipeline for image-based patient-specific analysis of cerebral aneurysm hemodynamics: technique and sensitivity. IEEE Trans Med Imaging 2005;24:457-67

20. de Rooij NK, Velthuis BK, Algra A, et al. Configuration of the circle of Willis, direction of flow, and shape of the aneurysm as risk factors for rupture of intracranial aneurysms. J Neurol 2009;256:45-50

21. Dhar S, Tremmel M, Mocco J, et al. Morphology parameters for intracranial aneurysm rupture risk assessment. Neurosurgery 2008; 63:185-96; discussion 196-97

22. Cebral JR, Raschi M. Suggested connections between risk factors of intracranial aneurysms: a review. Ann Biomed Eng 2013;41:1366-83

23. Beck J, Rohde S, el Beltagy M, et al. Difference in configuration of ruptured and unruptured intracranial aneurysms determined by biplanar digital subtraction angiography. Acta Neurochir (Wien) 2003; 145:861-65

24. Morita A, Kirino T, Hashi K, et al; UCAS Japan Investigators. The natural course of unruptured cerebral aneurysms in a Japanese cohort. $N$ Engl J Med 2012;366:2474-82

25. Schneiders JJ, Marquering HA, van den Berg R, et al. Rupture-associated changes of cerebral aneurysm geometry: high-resolution $3 \mathrm{D}$ imaging before and after rupture. AJNR Am J Neuroradiol 2014;35:1358-62

26. Rahman M, Ogilvy CS, Zipfel GJ, et al. Unruptured cerebral aneurysms do not shrink when they rupture: multicenter collaborative aneurysm study group. Neurosurgery 2011;68:155-60; discussion $160-61$

27. Baharoglu MI, Lauric A, Gao BL, et al. Identification of a dichotomy in morphological predictors of rupture status between sidewalland bifurcation-type intracranial aneurysms. J Neurosurg 2012; 116:871-81

28. Dempere-Marco L, Oubel E, Castro M, et al. CFD analysis incorporating the influence of wall motion: application to intracranial aneurysms. Med Image Comput Comput Assist Interv 2006;9(pt 2):438-45

29. Valen-Sendstad K, Steinman DA. Mind the gap: impact of computational fluid dynamics solution strategy on prediction of intracranial aneurysm hemodynamics and rupture status indicators. AJNR Am J Neuroradiol 2014;35:536-43

30. Sonobe M, Yamazaki T, Yonekura M, et al. Small unruptured intracranial aneurysm verification study: SUAVe study, Japan. Stroke 2010;41:1969-77

31. Ishibashi T, Murayama Y, Urashima M, et al. Unruptured intracranial aneurysms: incidence of rupture and risk factors. Stroke 2009; 40:313-16 